\title{
The Range of Approximate Unitary Equivalence Classes of Homomorphisms from AH-algebras
}

\author{
Huaxin Lin
}

\begin{abstract}
Let $C$ be a unital AH-algebra and $A$ be a unital simple $C^{*}$-algebra with tracial rank zero. It has been shown that two unital monomorphisms $\phi, \psi: C \rightarrow A$ are approximately unitarily equivalent if and only if

$$
[\phi]=[\psi] \text { in } K L(C, A) \text { and } \tau \circ \phi=\tau \circ \psi \text { for all } \tau \in T(A)
$$

where $T(A)$ is the tracial state space of $A$. In this paper we prove the following: Given $\kappa \in K L(C, A)$ with $\kappa\left(K_{0}(C)_{+} \backslash\{0\}\right) \subset K_{0}(A)_{+} \backslash\{0\}$ and with $\kappa\left(\left[1_{C}\right]\right)=\left[1_{A}\right]$ and a continuous affine map $\lambda: T(A) \rightarrow T_{\mathrm{f}}(C)$ which is compatible with $\kappa$, where $T_{\mathrm{f}}(C)$ is the convex set of all faithful tracial states, there exists a unital monomorphism $\phi: C \rightarrow A$ such that

$$
[\phi]=\kappa \text { and } \tau \circ \phi(c)=\lambda(\tau)(c)
$$

for all $c \in C_{\text {s.a. and }} \tau \in T(A)$. Denote by $\operatorname{Mon}_{a u}^{e}(C, A)$ the set of approximate unitary equivalence classes of unital monomorphisms. We provide a bijective map

$$
\Lambda: \operatorname{Mon}_{a u}^{e}(C, A) \rightarrow K L T(C, A)^{++}
$$

where $K L T(C, A)^{++}$is the set of compatible pairs of elements in $K L(C, A)^{++}$and continuous affine maps from $T(A)$ to $T_{\mathrm{f}}(C)$.

Moreover, we realized that there are compact metric spaces $X$, unital simple AF-algebras $A$ and $\kappa \in K L(C(X), A)$ with $\kappa\left(K_{0}(C(X))_{+} \backslash\{0\}\right) \subset K_{0}(A)_{+} \backslash\{0\}$ for which there is no homomorphism $h: C(X) \rightarrow A$ so that $[h]=\kappa$.
\end{abstract}

\section{Introduction}

Recall that an AH-algebra is a $C^{*}$-algebra which is an inductive limit of $C^{*}$-algebras $C_{n}$, where $C_{n}=P_{n} M_{r(n)}\left(C\left(X_{n}\right)\right) P_{n}$ for some finite $\mathrm{CW}$ complex $X_{n}$ and projections $P_{n} \in M_{r(n)}\left(C\left(X_{n}\right)\right)$. Note that every unital separable commutative $C^{*}$-algebra is an AH-algebra and every AFalgebra is an AH-algebra. It was shown in [11] (see also Theorem 3.6 of [13]) that two unital monomorphisms $\phi, \psi: C \rightarrow A$, where $A$ is a unital simple $C^{*}$-algebra with tracial rank zero, are approximately unitarily equivalent if and only if

$$
[\phi]=[\psi] \text { and } \tau \circ \phi(c)=\tau \circ \psi(c)
$$

for all $c \in C_{\text {s.a. }}$ and $\tau \in T(A)$. This result plays a role in the study of classification of amenable $C^{*}$-algebras, or otherwise known as the Elliott program. It also has applications in the study of dynamical systems both classical and non-commutative ones (see [11]). It is desirable to know the range of the approximately unitary equivalence classes of monomorphisms from a unital AH-algebra $C$ into a unital simple $C^{*}$-algebra with tracial rank zero. For example, one may ask if given any $\kappa \in K L(C, A)$ and any continuous affine map $\lambda: T(A) \rightarrow T(C)$ there exists a monomorphism $\phi$ such that $[\phi]=\kappa$ and $\tau \circ h(c)=\lambda(\tau)(c)$ for all $c \in C_{\text {s.a. }}$ and $\tau \in T(A)$. 
When $C$ is a finite CW complex, it was shown (see also a previous result of L. Li [6]) in [10] that, for any $\kappa \in K K(C, A)$ with $\kappa\left(K_{0}(C)_{+} \backslash\{0\}\right) \subset K_{0}(A)_{+} \backslash\{0\}$ and with $\kappa\left(\left[1_{C}\right]\right)=\left[1_{A}\right]$, there exists a unital monomorphism $\phi: C \rightarrow A$ such that $[\phi]=\kappa$. It should be noted that both conditions that $\kappa\left(\left[1_{C}\right]\right)=\left[1_{A}\right]$ and $\kappa\left(K_{0}(C) \backslash\{0\}\right) \subset K_{0}(A) \backslash\{0\}$ are necessary for the existence of such $\phi$. One of the earliest such results (concerning monomorphisms from $C\left(\mathbb{T}^{2}\right)$ into a unital simple AF-algebra) of this kind appeared in a paper of Elliott and Loring ([3] see also [2]). It was shown in [10] that the same result holds for the case that $C$ is a unital simple AH-algebra which has real rank zero, stable rank one and weakly unperforated $K_{0}(C)$. Therefore, it is natural to expect that it holds for general unital AH-algebras.

Let $C$ be the unitization of $\mathcal{K}$, the algebra of compact operators on $l^{2}$. Then it does not have a faithful tracial state. Consequently, it can not be embedded into any unital UHF-algebra, or any unital simple $C^{*}$-algebra which has at least one tracial state (It has been shown that a unital AH-algebra $C$ can be embedded into a unital simple AF-algebra if and only if $C$ admits a faithful tracial state -see [13]). This example at least suggests that for general unital AH-algebras, the problem is slightly more complicated than the first thought. Moreover, we note that to provide the range of approximately unitary equivalence classes of unital monomorphisms from $C$, we also need to consider the map $\lambda: T(A) \rightarrow T(C)$. Let $X$ be a compact metric space and let $C=C(X)$. Suppose that $h: C \rightarrow A$ is a unital monomorphism and suppose that $\tau \in T(A)$. Then $\tau \circ h$ induces a Borel probability measure on $X$. Suppose that $\kappa \in K L(C, A)$ is given. It is clear that not every measure $\mu$ can be induced by those $h$ for which $[h]=\kappa$. Thus, we should consider a compatible pair $(\kappa, \gamma)$ which gives a more complete information on $K$-theory than either $\kappa$ or $\gamma$ alone.

The main result of this paper is to show that if $C$ is a unital AH-algebra, $A$ is any unital simple $C^{*}$-algebra with tracial rank zero, $\kappa \in K L(C, A)^{++}$(see 2.3 below) with $\kappa\left(\left[1_{C}\right]\right)=\left[1_{A}\right]$ and $\lambda: T(A) \rightarrow T_{\mathrm{f}}(C)$, where $T_{\mathrm{f}}(C)$ is the convex set of faithful tracial states, which is a continuous affine map and is compatible with $\kappa$, there is indeed a unital monomorphism $\phi: C \rightarrow A$ such that

$$
[\phi]=\kappa \text { in } K L(C, A) \text { and } \phi_{T}=\lambda .
$$

We also show that the existence of $\lambda$ is essential to provide homomorphisms $\phi$. In fact, we find out that there are compact metric spaces $X$, unital simple AF-algebras $A$ and $\kappa \in$ $K L(C(X), A)^{++}$with $\left.\kappa\left(\left[1_{C}\right]\right)=\left[1_{A}\right]\right)$ for which there are no $\lambda: T(A) \rightarrow T_{\mathrm{f}}(C(X))$ which is compatible with $\kappa$. Moreover, we discovered that there are no homomorphism $h: C \rightarrow A$ (not just monomorphisms) such that $[h]=\kappa$. This further demonstrates that tracial information is an integral part of $K$-theoretical information.

\section{Notation}

2.1. Let $A$ be a unital $C^{*}$-algebra. Denote by $T(A)$ the tracial state space of $A$. Denote by $\operatorname{Aff}(T(A))$ the space of all real affine continuous functions on $T(A)$. If $\tau \in T(A)$, we will also use $\tau$ for the tracial state $\tau \otimes \operatorname{Tr}$ on $M_{k}(A)$ for all integer $k \geq 1$, where $\operatorname{Tr}$ is the standard trace on $M_{k}$. If $a \in A_{\text {s.a. }}$, denote by $\check{a}$ a real affine function in $\operatorname{Aff}(T(A))$ defined by $\check{a}(\tau)=\tau(a)$ for all $\tau \in T(A)$.

Let $C$ be another unital $C^{*}$-algebra. Suppose that $\gamma: \operatorname{Aff}(T(C)) \rightarrow \operatorname{Aff}(T(A))$ is a positive linear map. We say it is unital if $\gamma\left(1_{C}\right)(\tau)=1$. We say it is strictly positive, if $a \in \operatorname{Aff}(T(A))_{+} \backslash$ $\{0\}$, then $\gamma(a)(\tau)>0$ for all $\tau \in T(A)$.

Suppose that $\phi: C \rightarrow A$ is a unital homomorphism. Denote by $h_{T}: T(A) \rightarrow T(C)$ the affine continuous map induced by $h$, i.e.,

$$
h_{T}(\tau)(c)=\tau \circ h(c) \text { for all } c \in C .
$$


It also induces a positive linear map $h_{\sharp}: \operatorname{Aff}(T(C)) \rightarrow \operatorname{Aff}(T(A))$ defined by

$$
h_{\sharp}(\check{a})(\tau)=\tau \circ h(a) \text { for all } a \in C_{s . a} \text { and } \tau \in T(A),
$$

where $\check{a}(\tau)=\tau(a)$ for $\tau \in T(A)$.

If $\lambda: T(A) \rightarrow T(C)$ is an affine continuous map, then it gives a unital positive linear map $\lambda_{\sharp}: \operatorname{Aff}(T(C)) \rightarrow \operatorname{Aff}(T(A))$ by

$$
\lambda_{\sharp}(f)(\tau)=f(\lambda(\tau)) \text { for all } f \in \operatorname{Aff}(T(C)) \text { and for all } \tau \in T(A) .
$$

Conversely, a unital positive linear map $\gamma: \operatorname{Aff}(T(C)) \rightarrow \operatorname{Aff}(T(A))$ gives an affine continuous $\operatorname{map} \gamma_{T}: T(A) \rightarrow T(C)$ by

$$
f\left(\gamma_{T}(\tau)\right)=\gamma(f)(\tau) \text { for all } f \in \operatorname{Aff}(T(C)) \text { and } \tau \in T(C) .
$$

Suppose that $A$ is a unital simple $C^{*}$-algebra. Then $\gamma$ is strictly positive if and only if $\gamma_{T}$ maps $T(A)$ into $T_{\mathrm{f}}(C)$.

Denote by $\rho_{A}: K_{0}(A) \rightarrow \operatorname{Aff}(T(A))$ the positive homomorphism induced by $\rho_{A}([p])(\tau)=$ $\tau(p)$ for all projections $p \in M_{\infty}(A)$ and $\tau \in T(A)$.

Let $A$ and $C$ be two unital $C^{*}$-algebras and let $\kappa_{0}: K_{0}(C) \rightarrow K_{0}(A)$ be a unital positive homomorphism $\left(\kappa_{0}\left(\left[1_{C}\right]\right)=\left[1_{A}\right]\right)$. Suppose that $\lambda: T(A) \rightarrow T(C)$ is a continuous affine map. We say that $\lambda$ is compatible with $\kappa_{0}$, if $\tau(\kappa([p]))=\lambda(\tau)(p)$ for all projections $p$ in $M_{\infty}(A)$. Similarly, a unital positive linear map $\gamma: \operatorname{Aff}(T(C)) \rightarrow \operatorname{Aff}(T(A))$ is said to be compatible with $\kappa_{0}$, if $\gamma(\check{p})(\tau)=\tau\left(\kappa([p])\right.$ for all projections $p$ in $M_{\infty}(C) . \gamma$ is compatible with $\kappa_{0}$ if and only if $\gamma_{T}$ is so.

Two projections in $A$ are equivalent if there exists a partial isometry $w \in A$ such that $w^{*} w=p$ and $w w^{*}=q$.

2.2. Let $A$ be a unital $C^{*}$-algebra and let $C$ be a separable $C^{*}$-algebra which satisfies the universal coefficient theorem. By a result of Dadarlat and Loring ([1]),

$$
K L(C, A)=\operatorname{Hom}_{\Lambda}(\underline{K}(C), \underline{K}(A)),
$$

where, for any $C^{*}$-algebra $B$,

$$
\underline{K}(B)=\oplus_{i=0,1} K_{i}(B) \bigoplus_{n=2}^{\infty} \oplus_{i=0,1} K_{i}(B, \mathbb{Z} / n \mathbb{Z}) .
$$

We will identify two objects in (e2.1). Denote by

$$
\underline{K}_{F, k}(C)=\bigoplus_{i=0,1} K_{i}(B) \bigoplus_{n \mid k} \oplus_{i=0,1} K_{i}(B, \mathbb{Z} / n \mathbb{Z})
$$

If $K_{i}(C)$ is finitely generated $(i=0,1)$, then there is $k_{0} \geq 1$ such that

$$
\operatorname{Hom}_{\Lambda}(\underline{K}(C), \underline{K}(A)) \cong \operatorname{Hom}_{\Lambda}\left(F_{k_{0}} \underline{K}(C), F_{k_{0}} \underline{K}(A)\right)
$$

(see [1]).

Definition 2.3. Denote by $K L(C, A)^{++}$the set of those $\kappa \in \operatorname{Hom}_{\Lambda}(\underline{K}(C), \underline{K}(A))$ such that

$$
\kappa\left(K_{0}(C)_{+} \backslash\{0\}\right) \subset K_{0}(A) \backslash\{0\} .
$$

Denote by $K L_{e}(C, A)^{++}$the set of those $\kappa \in K L(C, A)^{++}$such that $\kappa\left(\left[1_{C}\right]\right)=\left[1_{A}\right]$. 
Definition 2.4. Let $\kappa \in K L_{e}(C, A)^{++}$and let $\lambda: T(A) \rightarrow T(C)$ be a continuous affine map. We say that $\lambda$ is compatible with $\kappa$ if $\lambda$ is compatible with $\left.\kappa\right|_{K_{0}(C)}$. Let $\gamma: \operatorname{Aff}(T(C)) \rightarrow \operatorname{Aff}(T(A))$ be a positive linear map. We say $\gamma$ is compatible with $\kappa$ if $\gamma$ is compatible with $\left.\kappa\right|_{K_{0}(C)}$, i.e., $\tau \circ \kappa([p])=\gamma(\check{p})(\tau)$ for all projections $p \in M_{\infty}(C)$.

2.5. Let $C=C(X)$ for some compact metric space $X$. One has the following short exact sequence:

$$
0 \rightarrow \operatorname{ker} \rho_{C} \rightarrow K_{0}(C) \rightarrow C(X, \mathbb{Z}) \rightarrow 0 .
$$

It is then easy to see that, for every projection $p \in M_{\infty}(C)$, there is a projection $q \in C$ and an integer $n$ such that $\rho_{A}([p])=n \rho_{A}([q])$. It follows that if $C$ is a unital AH-algebra, then for every projection $p \in M_{\infty}(C)$, there is a projection $q \in C$ and an integer $n \geq 1$ such that

$$
\rho_{A}([p])=n \rho_{A}([q]) .
$$

Note also that in this case $\operatorname{Aff}(T(C))=C_{s . a}$. Therefore, in this note, instead of considering a unital positive linear maps $\gamma: \operatorname{Aff}(T(C)) \rightarrow \operatorname{Aff}(T(A))$, we may consider unital positive linear maps $\gamma: C_{s . a} \rightarrow \operatorname{Aff}(T(A))$. Moreover, $\gamma$ is compatible with some $\kappa \in K L(C, A)^{++}$, if $\gamma(p)(\tau)=\tau(\kappa([p]))$ for all projections $p \in C$ and $\tau \in T(A)$.

2.6. Let $\phi, \psi: C \rightarrow A$ be two maps between $C^{*}$-algebras. Let $\epsilon>0$ and $\mathcal{F} \subset C$ be a subset. We write

$$
\phi \approx_{\epsilon} \psi \text { on } \mathcal{F}
$$

if

$$
\|\phi(c)-\psi(c)\|<\epsilon \text { for all } c \in \mathcal{F} .
$$

2.7. Let $L: C \rightarrow A$ be a linear map. Let $\delta>0$ and $\mathcal{G} \subset C$ be a (finite) subset. We say $L$ is $\delta$ - $\mathcal{G}$-multiplicative if

$$
\|L(a b)-L(a) L(b)\|<\delta \text { for all } a, b \in \mathcal{G} .
$$

Definition 2.8. Let $A$ be a unital $C^{*}$-algebra. Denote by $U(A)$ the unitary group of $A$. Let $B \subset A$ be another $C^{*}$-algebra and $\phi: B \rightarrow A$ be a map. We write $\phi=\operatorname{ad} u$ for some $u \in U(A)$ if $\phi(b)=u^{*} b u$ for all $b \in B$.

Let $\phi, \psi: C \rightarrow A$ be two maps. We say that $\phi$ and $\psi$ are approximately unitarily equivalent if there exists a sequence of unitaries $\left\{u_{n}\right\} \subset A$ such that

$$
\lim _{n \rightarrow \infty} \operatorname{ad} u_{n} \circ \phi(c)=\psi(c) \text { for all } c \in C .
$$

\section{Approximate unitary equivalence}

We begin with the following theorem

Theorem 3.1. (Theorem 3.6 of [13] and see also Theorem 3.4 of [11]) Let $C$ be a unital AHalgebra and let $A$ be a unital simple $C^{*}$-algebra with tracial rank zero. Suppose that $\phi, \psi: C \rightarrow A$ are two unital monomorphisms. Then there exists a sequence of unitaries $\left\{u_{n}\right\} \subset A$ such that

$$
\lim _{n \rightarrow \infty} \operatorname{ad} u_{n} \circ \psi(c)=\phi(c) \text { for all } c \in C,
$$

if and only if

$$
[\phi]=[\psi] \text { in } K L(C, A) \text { and } \tau \circ \phi=\tau \circ \psi \text { for all } \tau \in T(A) \text {. }
$$

We need the following variation of results in [1]. 
Theorem 3.2. Let $C$ be a unital $A H$-algebra, let $A$ be a unital simple $C^{*}$-algebra with $T R(A)=$ 0 and let $\gamma: C_{\text {s.a. }} \rightarrow \operatorname{Aff}(T(A))$ be a unital strictly positive linear map.

For any $\epsilon>0$ and any finite subset $\mathcal{F} \subset C$, there exist $\eta>0, \delta>0$, a finite subset $\mathcal{G} \subset C$, a finite subset $\mathcal{H} \subset C_{\text {s.a. }}$ and a finite subset $\mathcal{P} \subset \underline{K}(C)$ satisfying the following:

Suppose that $L_{1}, L_{2}: C \rightarrow A$ are two unital completely positive linear maps which are $\delta$ - $\mathcal{G}$ multiplicative such that

$$
\begin{aligned}
{\left.\left[L_{1}\right]\right|_{\mathcal{P}} } & =\left.\left[L_{2}\right]\right|_{\mathcal{P}} \\
\left|\tau \circ L_{i}(g)-\gamma(g)(\tau)\right| & <\eta \text { for all } g \in \mathcal{H}, \quad i=1,2
\end{aligned}
$$

Then there is a unitary $u \in A$ such that

$$
\operatorname{ad} u \circ L_{2} \approx_{\epsilon} L_{2} \text { on } \mathcal{F} \text {. }
$$

Proof. Write $C=\overline{\cup_{n=1}^{\infty} C_{n}}$, where $C_{n}=P_{n} M_{r(n)}\left(C\left(X_{n}\right)\right) P_{n}$, where $X$ is a compact subset of a finite CW complex and where $P_{n} \in M_{r(n)}\left(C\left(X_{n}\right)\right)$ is a projection. Let $\epsilon>0$ and a finite subset $\mathcal{F} \subset C$ be fixed. Without loss of generality, we may assume that $\mathcal{F} \subset C_{1}$. Let $\eta_{0}>0$ such that

$$
\left|f(x)-f\left(x^{\prime}\right)\right|<\epsilon / 8 \text { for all } f \in \mathcal{F},
$$

if $\operatorname{dist}\left(x, x^{\prime}\right)<\eta_{0}$. Let $\left\{x_{1}, x_{2}, \ldots, x_{m}\right\} \subset X$ be $\eta_{0} / 2$-dense in $X$. Suppose that $O_{i} \cap O_{j}=\emptyset$ if $i \neq j$, where

$$
O_{j}=\left\{x \in X: \operatorname{dist}\left(x, x_{j}\right)<\eta_{0} / 2 s\right\}, \quad j=1,2, \ldots, m
$$

for some integer $s \geq 1$.

Choose non-zero element $g_{j} \in\left(C_{1}\right)_{s . a}$ such that $0 \leq g_{j} \leq 1$ whose support lies in $O_{j}$, $j=1,2, \ldots, m$. Note such $g_{j}$ exists (by taking those in the center for example). Choose

$$
\sigma_{0}=\min \left\{\inf \left\{\gamma\left(g_{j}\right)(\tau): \tau \in T(A)\right\}: 1 \leq j \leq m\right\} .
$$

Since $\gamma$ is strictly positive, $\sigma_{0}>0$. Set $\sigma=\min \left\{\sigma_{0} / 2,1 / 2 s\right\}$. Then, by Corollary 4.8 of [11], such $\delta>0, \eta>0, \mathcal{G}, \mathcal{H}$ and $\mathcal{P}$ exists.

Lemma 3.3. Let $X$ be a compact metric space, let $A$ be a unital simple $C^{*}$-algebra with $T R(A)=0$ and let $\gamma: C(X)_{\text {s.a. }} \rightarrow \operatorname{Aff}(T(A))$ be a unital strictly positive linear map.

Then, for any $\epsilon>0$ and any $\mathcal{F} \subset C(X)$, there exists $\delta>0$, a finite subset $\mathcal{G} \subset C(X)_{\text {s.a. }}$, a set $S_{1}, S_{2}, \ldots, S_{n}$ of mutually disjoint clopen subsets with $\cup_{i=1}^{n} S_{i}=X$, satisfying the following:

For any two unital homomorphisms $\phi_{1}, \phi_{2}: C(X) \rightarrow p A p$ with finite dimensional range for some projection $p \in A$ with $\tau(1-p)<\delta$ such that

$$
\begin{aligned}
{\left[\phi_{1}\left(\chi_{S_{i}}\right)\right] } & =\left[\phi_{2}\left(\chi_{S_{i}}\right)\right] \text { in } K_{0}(A), i=1,2, \ldots, n, \\
\left|\tau \circ \phi_{1}(g)-\gamma(g)(\tau)\right| & <\delta \text { and } \\
\left|\tau \circ \phi_{2}(g)-\gamma(g)(\tau)\right| & <\delta
\end{aligned}
$$

for all $g \in \mathcal{G}$ and for all $\tau \in T(A)$, there exist a unitary $u \in U(p A p)$ such that

$$
\operatorname{ad} u \circ \phi_{1} \approx_{\epsilon} \phi_{2} \text { on } \mathcal{F} \text {. }
$$

Proof. This follows from 3.2 immediately. There is a sequence of finite CW complex $X_{n}$ such that $C(X)=\lim _{n \rightarrow \infty}\left(C\left(X_{n}\right), h_{n}\right)$, where each $h_{n}$ is a unital homomorphism. Fix $\epsilon>0$ and a finite subset $\mathcal{F} \subset C(X)$. Without loss of generality, we may assume that $\mathcal{F} \subset h_{K}\left(\mathcal{F}_{K}\right)$ for some integer $K \geq 1$ and a finite subset $\mathcal{F}_{K}$. 
Given any finite subset $\mathcal{P} \subset \underline{K}(C(X))$, one obtains a finite subset $\mathcal{Q}_{k} \subset \underline{K}\left(C\left(X_{k}\right)\right)$ such that $\left[h_{k}\right]\left(\mathcal{Q}_{k}\right)=\mathcal{P}$ for some $k \geq 1$. Let $p_{1}, p_{2}, \ldots, p_{n}$ be mutually orthogonal projections corresponding to the connected components of $X_{k}$. To simplify notation, without loss of generality, we may assume that $k=K$.

There are mutually disjoint clopen sets $S_{1}, S_{2}, \ldots, S_{n}$ of $X$ with $\cup_{i=1}^{n} S_{i}=X$ such that $h_{k}\left(p_{i}\right)=$ $\chi_{S_{i}}, i=1,2, \ldots, n$. Since $\phi$ and $\psi$ are homomorphisms with finite dimensional range, if

$$
\left[\phi\left(\chi_{S_{i}}\right)\right]=\left[\psi\left(\chi_{S_{i}}\right)\right] \text { in } K_{0}(A),
$$

then

$$
\left[\phi \circ h_{k}\right]=\left[\psi \circ h_{k}\right] \text { in } K L\left(C\left(X_{k}\right), A\right) .
$$

This, in particular, implies that

$$
\left.[\phi]\right|_{\mathcal{P}}=\left.[\psi]\right|_{\mathcal{P}} .
$$

This above argument shows that the lemma follows from 3.2

Definition 3.4. Let $X$ be a compact metric space which is a compact subset of some finite CW complex $Y$. Then there exists a decreasing sequence of finite CW complexes $X_{n} \subset Y$ such that

$$
X \subset X_{n} \text { and } \lim _{n \rightarrow \infty} \operatorname{dist}\left(X_{n}, X\right)=0 .
$$

Denote by $s_{m, n}: C\left(X_{m}\right) \rightarrow C\left(X_{n}\right)$ (for $n>m$ ) and $s_{n}: C\left(X_{n}\right) \rightarrow C(X)$ be the surjective homomorphisms induced by the inclusion $X_{n+1} \subset X_{n}$ and $X \subset X_{n}$, respectively.

Lemma 3.5. Let $Y$ be a finite $C W$ complex and $X \subset Y$ be a compact subset. For any $\epsilon>0$, any finite subset $\mathcal{F} \subset C(X)$, there exists a finite subset $\mathcal{P} \subset \underline{K}(C(X))$, an integer $k \geq 1$ and an integer $N \geq 1$ satisfying the following:

For any unital homomorphisms $\phi, \psi: C\left(X_{m}\right) \rightarrow A(m \geq k)$ for any unital simple $C^{*}$-algebra with $T R(A)=0$ for which

$$
\left.[\phi]\right|_{\mathcal{Q}}=\left.[\psi]\right|_{\mathcal{Q}} \text { in } K L\left(C\left(X_{m}\right), A\right),
$$

where $Q \subset \underline{K}\left(C\left(X_{m}\right)\right)$ is a finite subset such that $\left[s_{m}\right](\mathcal{Q})=\mathcal{P}$, then there exists a unitary $U \in M_{N+1}(A)$ such that

$$
a d U \circ\left(\phi \oplus \Phi \circ s_{m}\right) \approx_{\epsilon}\left(\psi \oplus \Phi \circ s_{m}\right) \text { on } s_{m}^{-1}(\mathcal{F}),
$$

where $\Phi: C(X) \rightarrow M_{N}(A)$ is defined by

$$
\Phi(f)=\operatorname{diag}\left(f\left(x_{1}\right), f\left(x_{2}\right), \ldots, f\left(x_{N}\right)\right) \text { for all } f \in C\left(X_{1}\right),
$$

where $\left\{x_{1}, x_{2}, \ldots, x_{N}\right\}$ is a finite subset of $X$.

Proof. Assume that the lemma were false. Then there would be a positive number $\epsilon_{0}>0$, a finite subset $\mathcal{F}_{0} \subset C(X)$, an increasing sequence of finite subsets $\left\{\mathcal{P}_{n}\right\} \subset \underline{K}(C(X))$ with $\cup_{n} \mathcal{P}_{n}=\underline{K}(C(X))$, a sequence of unital $C^{*}$-algebras, two subsequences $\{R(n)\},\{k(n)\}$ of $\mathbb{N}$ and two sequences monomorphisms $\phi_{n}, \psi_{n}: C\left(X_{k(n)}\right) \rightarrow A_{n}$ such that

$$
\begin{gathered}
{\left.\left[\phi_{n}\right]\right|_{\mathcal{Q}_{n}}=\left.\left[\psi_{n}\right]\right|_{\mathcal{Q}_{n}} \text { in } K K\left(C\left(X_{k(n)}\right), A_{n}\right) \text { and }} \\
\left.\underset{n}{\lim \sup }\left\{\inf \left\{\max \left\{\| u_{n}^{*}\left(\phi_{n} \oplus \Phi_{n} \circ s_{n}\right)(f)\right) u_{n}-\left(\phi \oplus \Phi_{n} \circ s_{n}\right)(f) \|: f \in s_{m}^{-1}(\mathcal{F})\right\}\right\}\right\} \geq \epsilon_{0},
\end{gathered}
$$

where infimum is taken among all possible $\Phi_{n}: C(X) \rightarrow M_{R(n)}\left(A_{n}\right)$ with the form described above and among all possible unitaries $\left\{u_{n}\right\} \subset U\left(M_{R(n)+1}(A)\right)$, and where $\mathcal{Q}_{n} \subset \underline{K}\left(C\left(X_{k(n)}\right)\right)$ 
is a finite subset such that $\left[s_{k(n)}\right]\left(\mathcal{Q}_{n}\right)=\mathcal{P}_{n}$. Since $K_{i}\left(C\left(X_{n}\right)\right.$ is finitely generated, by passing to a subsequence, if necessary, without loss of generality, we may assume (see also the end of 2.2) that

$$
\left[\phi_{n+1} \circ s_{k(n), k(n+1)}\right]=\left[\psi_{n+1} \circ s_{k(n), k(n+1)}\right] \text { in } K L\left(C\left(X_{k(n)}\right), A\right), \quad n=1,2, \ldots
$$

Let $\phi_{n}^{(m)}=\phi_{m}$, if $n \leq m, \phi_{n}^{(m)}=\phi_{n} \circ s_{m, n}, \psi_{n}^{(m)}=\psi_{m}$, if $n \leq m$ and $\psi_{n}^{(m)}=\psi_{n} \circ s_{m, n}, n=1,2, \ldots$ Denote by $H_{1}^{(m)}, H_{2}^{(m)}: C\left(X_{k(m)}\right) \rightarrow \prod_{n} A_{n}$ by $H_{1}^{(m)}(f)=\left\{\phi_{n}^{(m)}\right\}$ and $H_{2}^{(m)}(f)=\left\{\psi_{n}^{(m)}\right\}$. Let $\pi: \prod_{n} A_{n} \rightarrow \prod_{n} A_{n} / \bigoplus_{n} A_{n}$ be the quotient map. Then $\pi \circ H_{1}^{(m)}$ and $\pi \circ H_{2}^{(m)}$ both have spectrum $X$. Moreover, for each $i$, all $\pi \circ H_{i}^{(m)}$ gives the same homomorphism $F_{i}: C(X) \rightarrow$ $\prod_{n} A_{n} / \bigoplus_{n} A_{n}, i=1,2$.

Since $T R\left(A_{n}\right)=0, A_{n}$ has real rank zero, stable rank one, weakly unperforated $K_{0}\left(A_{n}\right)$, by Corollary 2.1 of [5] and (e 3.12)

$$
\left[H_{1}^{(m+1)} \circ s_{k(m), k(m+1)}\right]=\left[H_{2}^{(m+1)} \circ s_{k(m), k(m+1)}\right] \text { in } K L\left(C\left(X_{k(m)}\right), \prod_{n} A_{n}\right)
$$

It follows from Corollary 2.1 of [5] again that

$$
\left[F_{1}\right]=\left[F_{2}\right] \text { in } K L\left(C, \prod_{n} A_{n} / \bigoplus_{n} A_{n}\right) .
$$

It then follows from Theorem 1.1 and the Remark 1.1 of [5] that there is an integer $N \geq 1$ and a unitary $W \in U\left(M_{N+1}\left(\prod_{n} A_{n} / \bigoplus_{n} A_{n}\right)\right)$ such that

$$
\text { ad } W \circ\left(F_{2} \oplus H_{0}\right) \approx_{\epsilon_{0} / 2}\left(F_{1} \oplus H_{0}\right) \text { on } \mathcal{F}_{0},
$$

where $H_{0}: C(X) \rightarrow M_{N}\left(\prod_{n} A_{n} / \bigoplus_{n} A_{n}\right)$ is defined by $H_{0}(f)=\sum_{i=1}^{N} f\left(x_{i}\right) E_{i}$ for all $f \in C(X)$, $x_{i} \in X$ and $E_{i}=\operatorname{diag} \overbrace{0, \ldots, 0}^{i-1}, 1,0, \ldots, 0), i=1,2, \ldots, N$.

There is a unitary $\left\{W_{n}\right\} \in U\left(\prod_{n} A_{n}\right)$ such that $\pi\left(\left\{W_{n}\right\}\right)=W$. Then, for some sufficiently large $n$,

$$
W_{n}^{*} \operatorname{diag}\left(\phi_{n}(f), f\left(x_{1}\right), f\left(x_{2}\right), \ldots, f\left(x_{N}\right)\right) W_{n} \approx_{\epsilon_{0}}\left(\psi_{n}(f), f\left(x_{1}\right), f\left(x_{2}\right), \ldots, f\left(x_{N}\right)\right)
$$

on $\mathcal{F}_{0}$. This contradicts (e3.11).

Remark 3.6. There exists a positive number $\eta>0$ and integer $N_{1}>0$ which depend only on $\epsilon$ and $\mathcal{F}$ such that $\left\{x_{1}, x_{2}, \ldots, x_{N}\right\}$ and an integer $N$ can be replaced by any $\eta$-dense finite subset $\left\{\xi_{1}, \xi_{2}, \ldots, \xi_{N_{1}}\right\}$ and integer $N_{1}$.

From the proof, we also know that the assumption that $A$ has tracial rank zero can be replaced by much weaker conditions (see Corollary 2.1 of [5]). The main difference of 3.5 and results in [5] is that homomorphisms $\phi$ and $\psi$ are not assumed to be from $C(X)$ to $A$.

\section{Monomorphisms from $C(X)$}

Lemma 4.1. Let $X$ be a finite $C W$ complex and let $A$ be a unital simple $C^{*}$-algebra with real rank zero, stable rank one and weakly unperforated $K_{0}(A)$. Let $e_{1}, e_{2}, \ldots, e_{m} \in C(X)$ be mutually orthogonal projections corresponding to connected components of $X$.

Suppose that $\kappa \in K K(C(X), A)^{++}$with $\kappa\left(\left[1_{C(X)}\right]\right)=\left[1_{A}\right]$. Then, for any projection $p \in A$ and any unital homomorphism $\phi_{0}: C(X) \rightarrow(1-p) A(1-p)$ with finite dimensional range such 
that $\phi_{0}\left(\left[e_{i}\right]\right)<\kappa\left(\left[e_{i}\right]\right), i=1,2, \ldots, m$. Then there exists a unital monomorphism $\phi_{1}: C(X) \rightarrow p A p$ such that

$$
\left[\phi_{1}+\phi_{0}\right]=\kappa \text { in } K K(C(X), A) \text {. }
$$

Proof. Since $\sum_{i=1}^{m} \kappa\left(\left[e_{i}\right]\right)=\left[1_{A}\right]$ and $A$ has stable rank one, there are mutually orthogonal projections $p_{1}, p_{2}, \ldots, p_{m} \in A$ such that

$$
\sum_{i=1}^{m} p_{i}=1_{A} \text { and }\left[p_{i}\right]=\kappa\left(\left[e_{i}\right]\right), \quad i=1,2, \ldots, m
$$

From this it is clear that we may reduce the general case to the case that $X$ is connected.

So now we assume that $X$ is connected. Then it is easy to see that

$$
\kappa-\left[\phi_{0}\right] \in K K(C(X), A)^{++}
$$

and $\left(\kappa-\left[\phi_{0}\right]\right)\left(\left[1_{C(X)}\right]\right)=p$. It follows from Theorem 4.7 of [10] that there is a monomorphism $\phi_{1}: C(X) \rightarrow p A p$ such that

$$
[\phi]=\kappa-\left[\phi_{0}\right] .
$$

Lemma 4.2. Let $X$ a compact metric space and let $A$ be a unital simple $C^{*}$-algebra with tracial rank zero. Suppose that $\gamma: C(X)_{s, a} \rightarrow \operatorname{Aff}(T(A))$ is a unital strictly positive linear map. Let $S_{1}, S_{2}, \ldots, S_{n}$ be a set of mutually disjoint clopen subsets of $X$ with $\cup_{i=1}^{n} S_{i}=X$. Then for any $\delta>0$ and any finite subset $\mathcal{G} \subset C(X)_{\text {s.a }}$, there exists a projection $p \in A$ with $p \neq 1_{A}$ and a unital homomorphism $h: C(X) \rightarrow p A p$ with finite dimensional range such that

$$
\begin{aligned}
|\tau \circ h(g)-\gamma(g)(\tau)| & <\delta \text { for all } g \in \mathcal{G} \text { and } \tau \in T(A), \text { and } \\
\tau \circ h\left(\chi_{S_{i}}\right) & <\gamma\left(\xi_{S_{i}}\right)(\tau) \text { for all } \tau \in T(A),
\end{aligned}
$$

$i=1,2, \ldots, n$.

Proof. Put

$$
d=\min \left\{\delta, \min \left\{\inf \left\{\gamma\left(\chi_{S_{i}}\right)(\tau): \tau \in T(A)\right\}: 1 \leq i \leq n\right\}\right\} .
$$

Since $\gamma$ is strictly positive, $d>0$.

Let $\mathcal{G}_{0}=\mathcal{G} \cup\left\{\chi_{S_{1}}, \chi_{S_{2}}, \ldots, \chi_{S_{n}}\right\}$. It follows from 4.3 of [12] that there is a unital homomorphism $h_{0}: C(X) \rightarrow A$ with finite dimensional range such that

$$
|\tau \circ h(g)-\gamma(g)(\tau)|<d / 8 n \text { for all } g \in \mathcal{G}_{0}
$$

and for all $\tau \in T(A)$. In particular,

$$
\left|\tau \circ h\left(\chi_{S_{i}}\right)-\gamma\left(\chi_{S_{i}}\right)(\tau)\right|<d / 8 n \text { for all } \tau \in T(A)
$$

$i=1,2, \ldots, n$.

Since $\rho_{A}\left(K_{0}(A)\right)$ is dense in $\operatorname{Aff}(T(A))$, there exists a projection $p_{0} \in A$ such that

$$
d / 2 n<\tau\left(p_{0}\right)<d / n \text { for all } \tau \in T(A) .
$$

Note that $\tau\left(p_{0}\right)<\gamma\left(\chi_{S_{i}}\right)(\tau)$ for all $\tau \in T(A), i=1,2, \ldots, n$. Moreover, by (e 4.20),

$$
\tau \circ h\left(\chi_{S_{i}}\right)>\gamma\left(\xi_{S_{i}}\right)(\tau)-d / 8 n \geq d-d / 8 n>\tau\left(p_{0}\right) .
$$


for all $\tau \in T(A)$.

Write $h_{0}(f)=\sum_{k=1}^{m} f\left(x_{k}\right) e_{k}$ for all $f \in C(X)$, where $x_{k} \in X$ and $\left\{e_{1}, e_{2}, \ldots, e_{k}\right\}$ is a set of mutually orthogonal projections with $\sum_{k=1}^{m} e_{k}=1_{A}$.

Note that

$$
h_{0}\left(\xi_{S_{j}}\right)=\sum_{x_{k} \in S_{j}} e_{k}
$$

Therefore (by (e 4.22$)$ )

$$
\left[p_{0}\right] \leq\left[\sum_{x_{k} \in S_{j}} e_{k}\right]
$$

By Zhang's Riesz interpolation property (see [14]), there are projections $e_{k}^{\prime} \leq e_{k}$ such that

$$
\left[p_{0}\right]=\left[\sum_{k \in S_{j}} e_{k}^{\prime}\right]
$$

By Zhang's half projection theorem (see Theorem 1.1 of [15]), for each $k$, there is a projection $e_{k}^{\prime \prime} \leq e_{k}^{\prime}$ such that

$$
\left[e_{k}^{\prime \prime}\right]+\left[e_{k}^{\prime \prime}\right] \geq\left[e_{k}^{\prime}\right]
$$

Thus

$$
2\left[\sum_{\chi_{k} \in S_{i}} e_{k}^{\prime \prime}\right] \geq\left[p_{0}\right], \quad i=1,2, \ldots, n .
$$

Therefore (by (e4.21) and (e 4.20)

$$
\begin{aligned}
\tau\left(\sum_{x_{k} \in S_{i}}\left(e_{k}-e_{k}^{\prime \prime}\right)\right) & <\tau \circ h_{0}\left(\chi_{S_{i}}\right)-(1 / 2) \tau\left(p_{0}\right) \\
& <\tau \circ h\left(\chi_{S_{i}}\right)-d / 4 n \\
& <\gamma\left(\chi_{S_{i}}\right)(\tau)-d / 8 n \text { for all } \tau \in T(A) .
\end{aligned}
$$

Let $p=\sum_{k=1}^{m}\left(e_{k}-e_{k}^{\prime \prime}\right)$. Then clearly that $p \neq 1$. Moreover,

$$
\tau(1-p)<d / 4 \text { for all } \tau \in T(A) .
$$

Define $h(f)=\sum_{k=1}^{m} f\left(x_{k}\right)\left(e_{k}-e_{k}^{\prime \prime}\right)$ for all $f \in C(X)$. Then

$$
\left|\tau \circ h(f)-\tau \circ h_{0}(f)\right|<\tau\left(\sum_{k=1}^{m} e_{k}^{\prime \prime}\right)=\tau(1-p)<d / 4<\delta
$$

for all $\tau \in T(A)$.

Then, by (e4.28),

$$
\tau \circ h\left(\chi_{S_{i}}\right)<\gamma\left(\chi_{S_{i}}\right)(\tau) \text { for all } \tau \in T(A)
$$

Lemma 4.3. Let $X$ a compact metric space and let $A$ be a unital simple $C^{*}$-algebra with tracial rank zero. Suppose that $\gamma: C(X)_{s, a} \rightarrow \operatorname{Aff}(T(A))$ is a unital strictly positive linear map. Let $S_{1}, S_{2}, \ldots, S_{n}$ be a set of mutually disjoint clopen subsets of $X$ with $\cup_{i=1}^{n} S_{i}=X$. Then for any $\delta>0, \eta>0$, for any integer $N$ and any $\eta$-dense subset $\left\{x_{1}, x_{2}, \ldots, x_{N}\right\}$ of $X$ and any finite 
subset $\mathcal{G} \subset C(X)_{\text {s.a }}$, there exists a projection $p \in A$ with $p \neq 1_{A}$ and a unital homomorphism $h: C(X) \rightarrow p A p$ with finite dimensional range such that

$$
\begin{aligned}
|\tau \circ h(g)-\gamma(g)(\tau)| & <\delta \text { for all } g \in \mathcal{G} \text { and } \tau \in T(A), \text { and } \\
\tau \circ h\left(\chi_{S_{i}}\right) & <\gamma\left(\chi_{S_{i}}\right)(\tau) \text { for all } \tau \in T(A),
\end{aligned}
$$

$i=1,2, \ldots, n$,

$$
h(f)=\sum_{i=1}^{N} f\left(x_{i}\right) e_{i} \oplus h_{1}(f) \text { for all } f \in C(X),
$$

where $h_{1}: C(X) \rightarrow\left(1-\sum_{i=1}^{N} e_{i}\right) A\left(1-\sum_{i=1}^{N} e_{i}\right)$ is a unital homomorphism with finite dimensional range and $\left\{e_{1}, e_{2}, \ldots, e_{N}\right\}$ is a set of mutually orthogonal projections such that $\left[e_{i}\right]=\left[e_{1}\right] \geq$ $[1-p], i=1,2, \ldots, N$.

Proof. Let $N \geq 1$ and let $\eta$-dense subset $\left\{x_{1}, x_{2}, \ldots, x_{N}\right\}$ of $X$ be given. Let $\eta_{0}>0$ such that

$$
\left|f(x)-f\left(x^{\prime}\right)\right|<\delta / 4 \text { for all } f \in \mathcal{G},
$$

provided that $\operatorname{dist}\left(x, x^{\prime}\right)<\eta_{0}$.

Choose $\eta_{0}>\eta_{1}>0$ such that $B\left(x_{i}, \eta_{1}\right)$ intersects with one and only one $S_{i}$ among $\left\{S_{1}, S_{2}, \ldots, S_{n}\right\}$.

Choose, for each $i$, a non-zero function $f_{i} \in C(X)$ with $0 \leq f \leq 1$ whose support is in $B\left(x_{i}, \eta_{1} / 2\right)$. Put

$$
d_{0}=\min \left\{\inf \left\{\gamma\left(f_{i}\right)(\tau): \tau \in T(A)\right\}: 1 \leq i \leq N\right\} .
$$

So $d_{0}>0$. Put $\delta_{1}=\min \left\{\delta / 8, \delta_{0} / 4\right\}$ and put $\mathcal{G}_{1}=\mathcal{G} \cup\left\{1_{C(X)}\right\} \cup\left\{f_{i}: i=1,2, \ldots, N\right\}$.

Now applying 4.2. We obtain a projection $p \in A$ and a unital homomorphism $h_{0}: C(X) \rightarrow$ $p A p$ such that

$$
\begin{aligned}
\left|\tau \circ h_{0}(g)-\gamma(g)(\tau)\right| & <\delta_{1} \text { for all } g \in \mathcal{G}_{1} \text { and } \\
\tau \circ h_{0}\left(\chi_{S_{i}}\right) & <\gamma\left(\chi_{S_{i}}\right)(\tau)
\end{aligned}
$$

for all $\tau \in T(A), i=1,2, \ldots, n$. Since $1_{C(X)} \in \mathcal{G}_{1}$, by (e4.35),

$$
\tau(1-p)<\delta_{1}<\delta_{0} / 4 \text { for all } \tau \in T(A) .
$$

Write $h_{0}(f)=\sum_{j=1}^{L} f\left(\xi_{j}\right) q_{j}$ for all $f \in C(X)$, where $\xi_{j} \in X$ and $\left\{q_{1}, q_{2}, \ldots, q_{L}\right\}$ is a set of mutually orthogonal projections with $\sum_{j=1}^{L} q_{j}=p$.

Define

$$
e_{i}^{\prime}=\sum_{\xi_{j} \in B\left(x_{i}, \eta_{1} / 2\right)} q_{j}, \quad i=1,2, \ldots, N .
$$

It follows from (e 4.35) that, for each $i$,

$$
\begin{aligned}
\tau\left(e_{i}^{\prime}\right) & \geq \tau \circ h_{0}\left(f_{i}\right) \\
& >\gamma\left(f_{i}\right)(\tau)-\delta_{1}>3 \delta_{0} / 4 \geq \tau(p)
\end{aligned}
$$

for all $\tau \in T(A)$. It follows that

$$
\left[e_{i}^{\prime}\right] \geq[p], \quad i=1,2, \ldots, N
$$


There are projections $e_{i} \leq e_{i}^{\prime}$ such that

$$
\left[e_{i}\right]=\left[e_{1}\right] \geq[1-p], \quad i=1,2, \ldots, N .
$$

Define

$$
\begin{aligned}
h_{1}(f) & =\sum_{\xi_{j} \notin \cup_{i=1}^{N} B\left(x_{i}, \eta_{1} / 2\right)} f\left(\xi_{j}\right) q_{j}+\sum_{i=j}^{N} f\left(x_{j}\right)\left(e_{i}^{\prime}-e_{i}\right) \text { and } \\
h(f) & =\sum_{i=1}^{N} f\left(x_{i}\right) e_{i} \oplus h_{1}(f)
\end{aligned}
$$

for all $f \in C(X)$. Since $B\left(x_{j}, \eta_{1} / 2\right)$ lies in one of $S_{i}$,

$$
\tau \circ h\left(\chi_{S_{i}}\right)=\tau \circ h_{0}\left(\chi_{S_{i}}\right) \text { for all } \tau \in T(A),
$$

$i=1,2, \ldots, n$. It follows from (e 4.36) that (e 4.32) holds. By the choice of $\eta_{0}$, we also have

$$
\left\|h_{0}(g)-h(g)\right\|<\delta / 2 \text { for all } h \in \mathcal{G} .
$$

Thus, by (e e.35), (e 4.31) also holds.

Lemma 4.4. Let $X$ be a compact metric space and let $A$ be a unital simple $C^{*}$-algebra with tracial rank zero. Suppose that $\gamma: C(X)_{\text {s.a }} \rightarrow \operatorname{Aff}(T(A))$ is a unital strictly positive linear map which is compatible with a strictly positive homomorphism $\kappa_{0}: K_{0}(C(X)) \rightarrow K_{0}(A)$. Fix $\delta>0$, $\eta>0$, a finite subset $\mathcal{F} \subset C(X)_{\text {s.a. }}$, an integer $N \geq 1$, an $\eta$-dense subset $\left\{x_{1}, x_{2}, \ldots, x_{N}\right\}$ of $X, a$ finitely many mutually disjoint clopen subset $S_{1}, S_{2}, \ldots, S_{n} \subset X$ with $\cup_{i=1}^{n} S_{i}=X$, a finite subset set $\left\{a_{1}, a_{2}, \ldots, a_{n}\right\} \subset A$ of mutually orthogonal projections with

$$
\left.0<a_{i}<\kappa_{0}\left(\left[\chi_{S_{i}}\right)\right]\right), \quad i=1,2, \ldots, n,
$$

a finitely many mutually disjoint clopen subsets $\left\{F_{1}, F_{2}, \ldots, F_{n_{1}}\right\}$ of $X$ with $\cup_{i=1}^{n_{1}} F_{i}=X$, and a projection $p$ with $\tau(p)=\tau\left(\sum_{i=1}^{n} a_{i}\right)$ for all $\tau \in T(A)$.

There is a projection $q \in A$ such that $[p] \leq[q]$ and a unital homomorphism $h: C(X) \rightarrow q A q$ with finite dimensional range such that

$$
\begin{aligned}
|\tau \circ h(g)-\gamma(g)(\tau)| & <\delta \text { for all } g \in \mathcal{F} \text { and } \tau \in T(A), \text { and } \\
\tau \circ h\left(\chi_{F_{i}}\right) & <\gamma\left(\chi_{F_{i}}\right)(\tau) \text { for all } \tau \in T(A),
\end{aligned}
$$

$i=1,2, \ldots, n$,

$$
h(f)=\sum_{i=1}^{N} f\left(x_{i}\right) e_{i} \oplus h_{1}(f) \text { for all } f \in C(X),
$$

where $h_{1}: C(X) \rightarrow\left(1-\sum_{i=1}^{N} e_{i}\right) A\left(1-\sum_{i=1}^{N} e_{i}\right)$ is a unital homomorphism with finite dimensional range and $\left\{e_{1}, e_{2}, \ldots, e_{N}\right\}$ is a set of mutually orthogonal projections such that $\left[e_{i}\right]=\left[e_{1}\right] \geq$ $[1-p], i=1,2, \ldots, N$.

Moreover, there exists a projection $p^{\prime} \in q$ such that

$$
\begin{gathered}
p^{\prime} h(f)=h(f) p^{\prime} \text { for all } f \in C(X) \text { and } \\
{\left[h\left(\chi_{S_{j}}\right) p^{\prime}\right]=\left[a_{j}\right], \quad j=1,2, \ldots, n .}
\end{gathered}
$$


Proof. Let

$$
\left.d_{0}=\min \left\{\inf \left\{\tau\left(\kappa_{0}\left(\left[\chi_{S_{i}}\right)\right]\right)-\left[a_{i}\right]\right): \tau \in T(A)\right\}: 1 \leq i \leq n\right\}
$$

and let

$$
d_{1}=\inf \{\tau(1-p): \tau \in T(A)\} .
$$

Then $d_{0}, d_{1}>0$. Define $\delta_{1}=\min \left\{\delta / 4, d_{0} / 2, d_{1} / 2\right\}$ and $\mathcal{G}_{1}=\mathcal{F} \cup\left\{1_{C(X)}, \chi_{S_{i}}, i=1,2, \ldots, n\right\}$. By applying 4.3, we obtain a projection $q \in A$ and a unital homomorphism $h: C(X) \rightarrow q A q$ with finite dimensional range satisfying the following:

$$
\begin{aligned}
|\tau \circ h(g)-\gamma(g)(\tau)| & <\delta_{1} \text { for all } g \in \mathcal{G}_{1}, \\
\tau \circ h\left(\chi_{F_{j}}\right) & <\gamma\left(\chi_{F_{j}}\right)(\tau)
\end{aligned}
$$

for all $\tau \in T(A), j=1,2, \ldots, n_{1}$,

$$
h(f)=\sum_{k=1}^{N} f\left(x_{k}\right) e_{i} \oplus h_{1}(f) \text { for all } f \in C(X),
$$

where $\left\{e_{1}, e_{2}, \ldots, e_{N}\right\}$ is a set of mutually orthogonal and mutually equivalent projections such that $\left[e_{1}\right] \geq[1-q]$, and where $h_{1}: C(X) \rightarrow\left(q-\sum_{k=1}^{N} e_{k}\right) A\left(q-\sum_{k=1}^{N} e_{k}\right)$ is a unital homomorphism with finite dimensional range.

Since $1_{C(X)} \in \mathcal{G}_{1}$, by the choice of $\delta_{1}$, we conclude that $[p] \leq[q]$.

Moreover, by (e 4.49),

$$
\tau \circ h\left(\chi_{S_{i}}\right)>\tau\left(a_{i}\right) \text { for all } \tau \in T(A), i=1,2, \ldots, n .
$$

Write

$$
h(f)=\sum_{s=1}^{L} f\left(\xi_{s}\right) E_{s} \text { for all } f \in C(X),
$$

where $\xi_{s} \in X$ and $\left\{E_{1}, E_{2}, \ldots, E_{L}\right\}$ is a set of mutually orthogonal projections such that $\sum_{s=1}^{L} E_{s}=$ $q$. By (e 4.52$)$, one has

$$
\sum_{\xi_{s} \in S_{i}} E_{s} \geq a_{i}, \quad i=1,2, \ldots, n
$$

For each $i$, by the Riesz Interpolation Property ([14]), there is a projection $E_{s}^{\prime} \leq E_{s}$ for which $x_{s} \in S_{i}$ such that

$$
\left[\sum_{\xi_{s} \in S_{i}} E_{s}^{\prime}\right]=\left[a_{i}\right]
$$

Put $p^{\prime}=\sum_{s=1}^{L} E_{s}^{\prime}$ Then

$$
\begin{gathered}
p^{\prime} h(f)=h(f) p^{\prime} \text { for all } f \in C(X) \text { and } \\
{\left[h\left(\chi_{S_{i}}\right) p^{\prime}\right]=\left[a_{i}\right], \quad i=1,2, \ldots, n .}
\end{gathered}
$$

Theorem 4.5. Let $X$ be a compact subset of a finite $C W$ complex and let $A$ be a unital simple $C^{*}$-algebra with $T R(A)=0$. Suppose that $\kappa \in K L_{e}(C(X), A)^{++}$and suppose that there exists a unital strictly positive linear map $\gamma: C(X)_{s . a} \rightarrow \operatorname{Aff}(T(A))$ which is compatible with $\kappa$. Then there exists a unital monomorphism $\phi: C(X) \rightarrow A$ such that

$$
[\phi]=\kappa \text { in } K L(C, A) \text {. }
$$


Proof. Suppose that $X \subset Y$, where $Y$ is a finite CW complex. Let $X_{n} \subset Y$ be a decreasing sequence of finite CW complexes for which 3.4 holds. Suppose that $p_{n, 1}, p_{n, 2}, \ldots, p_{n, r(n)}$ are mutually orthogonal projections of $C\left(X_{n}\right)$ which correspond to the connected components of $X_{n}$. It is clear that we may assume that each connected component of $X_{n}$ contains at least one point of $X$. This implies that $\left[s_{n}\right] \in K K\left(C\left(X_{n}\right), C(X)\right)^{++}$. It follows that

$$
\left[s_{n}\right] \times \kappa \in K K\left(C\left(X_{n}\right), A\right)^{++} .
$$

Let $\left\{\mathcal{F}_{n}\right\}$ be an increasing sequence of finite subsets of $C(X)$ whose union is dense in $C(X)$. Let $\left\{\eta_{n}\right\}$ be a decreasing sequence of positive numbers with $\lim _{n \rightarrow \infty} \eta_{n}=0,\left\{\mathcal{P}_{n}\right\}$ be an increasing sequence of finite subsets of $\underline{K}(C)$ whose union is $\underline{K}(C)$, let $\{k(n)\},\{N(n)\} \subset \mathbb{N}$ be two sequences of integers such that $k(n), N(n) \nearrow \infty$, and $\left\{x_{n, 1}, x_{(n, 2}, \ldots, x_{n, N(n)}\right\}$ be $\eta_{n}$-dense subsets of $X$ which satisfy the requirements of 3.5 and 3.6 for corresponding $\epsilon_{n}=1 / 2^{n+2}$ and $\mathcal{F}_{n}$.

By passing to a subsequence if necessary, we may assume that there is a finite subset $\mathcal{F}_{n}^{\prime} \subset$ $C\left(X_{k(n+1)}\right)$ such that $s_{k(n+1)}\left(\mathcal{F}_{n}^{\prime}\right)=\mathcal{F}_{n}$ and a finite subset $\mathcal{Q}_{k(n)} \subset \underline{K}\left(C\left(X_{k(n)}\right)\right)$ such that $\left[s_{k(n)}\right]\left(\mathcal{Q}_{k(n)}\right)=\mathcal{P}_{n}, n=1,2, \ldots$ We may assume that $1_{C\left(X_{k(n)}\right)} \in \mathcal{F}_{n}^{\prime}$, without loss of generality.

Set $\kappa_{n}=\left[s_{k(n)}\right] \times \kappa$. Note that $\kappa_{n}\left(\left[1_{C\left(X_{k(n)}\right)}\right]\right)=\left[1_{A}\right]$.

Let $\delta_{n}$ (in place of $\delta$ ), $\mathcal{G}_{n}^{\prime} \subset C(X)_{s . a}$ ( in place of $\mathcal{G}$ ), $S_{1, n}, S_{2, n}, \ldots, S_{m(n), n}$ (in place of $\left.\left\{S_{1}, S_{2}, \ldots,\right\}\right)$ be a set of disjoint clopen subsets of $X$ with $\cup_{i=1}^{m(n)} S_{i}=X$ required by 3.3 for $\epsilon_{n}$ and $\mathcal{F}_{n}, n=1,2, \ldots$. We may assume that $1_{C(X)} \in \mathcal{G}_{n}^{\prime}, n=1,2, \ldots$.

By taking a refinement of the clopen partition of $X$, we may assume that $s_{n}\left(p_{n, i}\right)$ is a finite sum of functions in $\left\{\chi_{S_{j, n}}: 1 \leq j \leq m(n)\right\}, i=1,2, \ldots, r(n)$.

Let $\mathcal{G}_{n} \subset C\left(X_{k(n)}\right)_{s . a}$. be a finite subsets for which $s_{k(n)}\left(\mathcal{G}_{n}\right)=\mathcal{G}_{n}^{\prime}, n=1,2, \ldots$.

By applying 4.3, we obtain a projection $P_{1} \in A$ and a unital homomorphism $\Phi_{1}^{\prime}: C(X) \rightarrow$ $P_{1} A P_{1}$ such that

$$
\begin{aligned}
\left|\tau \circ \Phi_{1}^{\prime}(g)-\gamma(g)(\tau)\right| & <\delta_{1} / 2 \text { for all } g \in \mathcal{G}_{1}^{\prime}, \\
\tau \circ \Phi_{1}^{\prime}\left(\chi_{S_{j, 1}}\right) & <\gamma\left(\chi_{S_{j, 1}}\right)(\tau)
\end{aligned}
$$

for all $\tau \in T(A), i=1,2, \ldots, m(1)$, and

$$
\Phi_{1}^{\prime}(f)=\sum_{i=1}^{N(1)} f\left(x_{1, i}\right) e_{i}^{(1)} \oplus \Phi_{0,1}^{\prime}(f) \text { for all } f \in C(X),
$$

where $\left\{e_{1}^{(1)}, e_{2}^{(1)}, \ldots, e_{N(1)}^{(1)}\right\}$ is a set of mutually orthogonal and mutually equivalent projections with $\left[e_{1}\right] \geq\left[\left(1-P_{1}\right)\right]$ and where $\Phi_{0,1}^{\prime}: C(X) \rightarrow\left(P_{1}-\sum_{i=1}^{N(1)} e_{i}^{(1)}\right) A\left(\left(P_{1}-\sum_{i=1}^{N(1)} e_{i}^{(1)}\right)\right.$ is a unital homomorphism with finite dimensional range. Note also, since $1_{C(X)} \in \mathcal{G}_{1}^{\prime}, \tau\left(1-P_{1}\right)<\delta_{1} / 2$ for all $\tau \in T(A)$.

It follows from 4.1 that there is a unital monomorphism $\phi_{1}^{\prime}: C\left(X_{k(1)}\right) \rightarrow\left(1-P_{1}\right) A\left(1-P_{1}\right)$ such that

$$
\left[\phi_{1}^{\prime}\right]+\left[\Phi_{1}^{\prime} \circ s_{1}\right]=\kappa_{1} \text { in } K K\left(C\left(X_{k(1)}\right), A\right) .
$$

Define $\phi_{1}=\phi_{1}^{\prime}+\Phi_{1}^{\prime} \circ s_{1}$.

Suppose that, for $1 \leq m \leq n$, there are unital homomorphisms $\phi_{m}^{\prime}: C\left(X_{k(m)}\right) \rightarrow(1-$ $\left.P_{m}\right) A\left(1-P_{m}\right)$ and $\Phi_{m}^{\prime}: C(X) \rightarrow P_{m} A P_{m}$ and a unital (injective) homomorphism $\phi_{m}=$ $\phi_{m}^{\prime}+\Phi_{m}^{\prime} \circ s_{k(m)}$ such that 
(1) there are mutually orthogonal and mutually equivalent projections $e_{1}^{(m)}, e_{2}^{(m)}, \ldots, e_{N(m)}^{(m)} \in$ $P_{m} A P_{m}$ for which $\left[e_{1}^{(m)}\right] \geq\left[1-P_{m}\right]$, and

$$
\Phi_{m}^{\prime}(f)=\sum_{i=1}^{N(m)} f\left(x_{m, i}\right) e_{i}^{(m)} \oplus \Phi_{m}^{(0)}(f) \text { for all } f \in C(X)
$$

where $\Phi_{m}^{(0)}: C(X) \rightarrow\left(P_{m}-\sum_{i=1}^{N(m)} e_{i}^{(m)}\right) A\left(P_{m}-\sum_{i=1}^{N(m)} e_{i}^{(m)}\right)$ is a unital homomorphism with finite dimensional range;

(2) $\tau \circ \Phi^{\prime}\left(\chi_{S_{j, m}}\right)<\gamma\left(\chi_{S_{j, m}}\right)(\tau)$ for all $\tau \in T(A), j=1,2, \ldots, m(m)$;

(3) $\left|\tau \circ \Phi_{m}^{\prime}(g)-\gamma(g)(\tau)\right|<\delta_{m} / 2$ for all $g \in \mathcal{G}_{m}^{\prime}$ and for all $\tau \in T(A)$;

(4) $\left[P_{m+1}\right] \geq\left[P_{m}\right]$ in $K_{0}(A)$ and $\tau\left(1-P_{m}\right)<\delta_{m} / 2$ for all $\tau \in T(A)$;

(5) there is a projection $P_{m+1}^{\prime} \leq P_{m+1}$ such that $P_{m+1}^{\prime} \Phi_{m+1}=\Phi_{m+1}^{\prime} P_{m+1}$ and

$$
\left[\Phi_{m+1}^{\prime}\left(\chi_{S_{j, m}}\right) P_{m+1}^{\prime}\right]=\left[\Phi_{m}^{\prime}\left(\chi_{S_{j, m}}\right)\right] \text { in } K_{0}(A), j=1,2, \ldots, m(m) \text {; }
$$

(6) $\phi_{m}^{\prime}$ is a unital monomorphism;

(7) $\left[\phi_{m}\right]=\left[\phi_{m}^{\prime}\right]+\left[\Phi_{m}^{\prime} \circ s_{k(m)}\right]=\kappa_{m}$;

(8) there exists a unitary $u_{m} \in A$ such that

$$
\text { ad } u_{m} \circ \phi_{m+1} \circ s_{k(m), k(m+1)} \approx_{1 / 2^{m+1}} \phi_{m} \text { on } s_{k(m)}^{-1}\left(\mathcal{F}_{m}\right), m=1,2, \ldots, n-1 \text {. }
$$

It follows from 4.4 that there is a projection $P_{n+1} \in A$ and a unital homomorphism $\Phi_{n+1}^{\prime}$ : $C(X) \rightarrow P_{n+1} A P_{n+1}$ satisfying the following:

(1) there are mutually orthogonal and mutually equivalent projections $e_{1}^{(n+1)}, e_{2}^{(n+1)}, \ldots, e_{N(n+1)}^{(n+1)} \in$ $P_{n+1} A P_{n+1}$ for which $\left[e_{1}^{(n+1)}\right] \geq\left[1-P_{n+1}\right]$, and

$$
\Phi_{n+1}^{\prime}(f)=\sum_{i=1}^{N(n+1)} f\left(x_{n+1, i}\right) e_{i}^{(n+1)} \oplus \Phi_{n+1}^{(0)}(f) \text { for all } f \in C(X)
$$

where $\Phi_{n+1}^{(0)}: C(X) \rightarrow\left(P_{n+1}-\sum_{i=1}^{N(n+1)} e_{i}^{(n+1)}\right) A\left(P_{n+1}-\sum_{i=1}^{N(n+1)} e_{i}^{(n+1)}\right)$ is a unital homomorphism with finite dimensional range;

(2) $\tau \circ \Phi_{n+1}^{\prime}\left(\chi_{S_{j, n+1}}\right)<\gamma\left(\chi_{S_{j, n+1}}\right)(\tau)$ for all $\tau \in T(A), j=1,2, \ldots, m(n+1)$;

(3) $\left|\tau \circ \Phi_{n+1}^{\prime}(g)-\gamma(g)(\tau)\right|<\delta_{n+1} / 2$ for all $g \in \mathcal{G}_{n+1}^{\prime}$ and for all $\tau \in T(A)$;

(4) $\left[P_{n+1}\right] \geq\left[P_{n}\right]$ in $K_{0}(A)$ and $\tau\left(1-P_{n+1}\right)<\delta_{n+1} / 2$ for all $\tau \in T(A)$;

(5) there is a projection $P_{n+1}^{\prime} \leq P_{n+1}$ such that $P_{n+1}^{\prime} \Phi_{n+1}^{\prime}=\Phi_{n+1}^{\prime} P_{n+1}$ and

$$
\left[\Phi_{n+1}^{\prime}\left(\chi_{S_{n, j}}\right) P_{n+1}^{\prime}\right]=\left[\Phi_{n}^{\prime}\left(\chi_{S_{n, j}}\right)\right] \text { in } K_{0}(A), j=1,2, \ldots, m(n) .
$$


It follows from 4.1 that there is a unital monomorphism $\phi_{n+1}^{\prime}: C\left(X_{k(n+1)}\right) \rightarrow\left(1-P_{n+1}\right) A\left(1-P_{n+1}\right)$ such that

$$
\left[\phi_{n+1}^{\prime}\right]=\kappa_{n+1}-\left[\Phi_{n+1}^{\prime} \circ s_{k(n+1)}\right] \text { in } K K\left(C\left(X_{k(n+1)}, A\right)\right.
$$

Define $\phi_{n+1}=\phi_{n+1}^{\prime}+\Phi_{n+1}^{\prime} \circ s_{k(n+1)}$.

Thus $\phi_{n+1}^{\prime}, \phi_{n+1}^{\prime}$ and $\phi_{n+1}$ satisfy (1), (2), (3), (4), (5), (6) and (7).

To complete the induction, define $\Phi_{n+1}^{\prime \prime}: C(X) \rightarrow P_{n+1}^{\prime} A P_{n+1}^{\prime}$ by $\Phi_{n+1}^{\prime \prime}(f)=P_{n+1}^{\prime} \Phi_{n+1}^{\prime}(f) P_{n+1}^{\prime}$ for all $f \in C(X)$. By (3) and (4),

$$
\left|\tau \circ \Phi_{n+1}^{\prime \prime}(g)-\gamma(g)(\tau)\right|<\delta_{n+1} / 2 \text { for all } g \in \mathcal{G}_{n}
$$

for all $\tau \in T(A)$. Note that, by $(5),\left[P_{n+1}^{\prime}\right]=\left[P_{n}\right]$. There is a unitary $w_{n} \in U(A)$ such that

$$
w_{n}^{*} P_{n+1}^{\prime} w_{n}=P_{n} .
$$

Thus, by (5) and (3), and by applying [3.3. there exists a unitary $v_{n} \in U\left(P_{n} A P_{n}\right)$ such that

$$
\operatorname{ad} v_{n} \circ \operatorname{ad} w_{n} \circ \Phi_{n+1}^{\prime \prime} \approx_{\epsilon_{n}} \Phi_{n}^{\prime} \text { on } \mathcal{F}_{n} .
$$

Denote $\Psi_{n+1}^{\prime}=P_{n+1}^{\prime} \Phi_{n+1} P_{n+1}^{\prime}$ and $\Psi_{n+1}=\operatorname{ad} w_{n} \circ \Psi_{n+1}^{\prime}$. Let $\phi_{n+1}^{\prime \prime}=\operatorname{ad} w_{n} \circ \phi_{n+1}^{\prime} \oplus \Psi_{n+1}$. Now consider $\phi_{n}^{\prime}$ and $\phi_{n+1}^{\prime \prime} \circ s_{k(n), k(n+1)}$. By (7) and (e 4.62), we have

$$
\left.\left[\phi_{n+1}^{\prime \prime} \circ s_{k(n), k(n+1)}\right]\right|_{\mathcal{Q}_{k(n)}}=\left.\left[\phi_{n}^{\prime}\right]\right|_{\mathcal{Q}_{k(n)}} .
$$

It follows from (1) and 3.5 that there exists a unitary $V_{n} \in U(A)$ such that

$$
\text { ad } V_{n} \circ\left(\phi_{n+1}^{\prime \prime} \circ s_{k(n), k(n+1)} \oplus \Phi_{n}^{\prime} \circ s_{k(n)}\right) \approx_{\epsilon_{n}} \phi_{n}^{\prime} \oplus \Phi_{n}^{\prime} \circ s_{k(n)} \text { on } s_{k(n)}^{-1}\left(\mathcal{F}_{n}\right) \text {. }
$$

Define $u_{n}=w_{n}\left(v_{n}+\left(1-P_{n}\right)\right) V_{n}$. Then, by (e4.63) and (e4.64),

$$
\operatorname{ad} u_{n} \circ \phi_{n+1} \approx_{2 \epsilon_{n}} \phi_{n} \text { on } s_{k(n)}^{-1}\left(\mathcal{F}_{n}\right) \text {. }
$$

Note $2 \epsilon_{n}=1 / 2^{n+1}$.

This concludes the induction.

Define $\psi_{1}=\phi_{1}$ and $\psi_{n+1}=\operatorname{ad} u_{n} \circ \phi_{n+1}, n=1,2, \ldots$ Then, by (8) above,

$$
\left\|\psi_{n}(c)-\psi_{n+1} \circ s_{k(n), k(n+1)}(c)\right\|<1 / 2^{n+2} \text { for all } c \in s_{k(n)}^{-1}\left(\mathcal{F}_{n}\right),
$$

$n=1,2, \ldots$

Fix $m$ and $f \in \mathcal{F}_{m}$, let $g \in s_{k(m)}^{-1}\left(\mathcal{F}_{m}\right)$ such that $s_{k(m)}(g)=f$.

It follows that $\left\{\psi_{n} \circ s_{m, n}(g)\right\}_{n \geq m}$ is a Cauchy sequence by (e4.66).

Note that if $g^{\prime} \in s_{k(m)}^{-1}\left(\mathcal{F}_{m}\right)$ such that $s_{k(m)}(g)=s_{k(m)}\left(g^{\prime}\right)$, then, for any $\epsilon>0$, there exists $n \geq m$ such that

$$
\left\|s_{k(m), k(n)}(g)-s_{k(m), k(n)}\left(g^{\prime}\right)\right\|<\epsilon .
$$

Thus $h(f)=\lim _{n \rightarrow \infty} \psi_{n} \circ s_{m, n}(g)$ is well-defined. It is then easy to verify that $h$ defines a unital homomorphism from $C(X)$ into $A$. Since each $\phi_{n}$ is injective, it is easy to check that $h$ is also injective.

If $x \in \mathcal{Q}_{m}$, then by (7) above,

$$
[h] \circ\left[s_{k(m)}\right](x)=\kappa_{n} \circ\left[s_{k(m), k(n)}\right](x)=\kappa \circ\left[s_{k(m)}\right](x) .
$$

Therefore

$$
[h]=\kappa \text { in } K L(C, A) .
$$

It is also easy to check from (3) and (4) that

$$
\tau \circ h(g)=\gamma(\check{g})(\tau) \text { for all } g \in C(X)_{s . a}
$$

and for all $\tau \in T(A)$. 


\section{AH-algebras}

Lemma 5.1. Let $X$ be a compact subset of a finite $C W$ complex, let $C=P M_{k}(C(X)) P$, where $P \in M_{k}(C(X))$ is a projection, and let $A$ be a unital simple $C^{*}$-algebra with tracial rank zero. Suppose that $\kappa \in K L_{e}(C, A)^{++}$and suppose that $\gamma: C_{\text {s.a. }} \rightarrow \operatorname{Aff}(T(A))$ is a unital positive linear map which is compatible with $\kappa$. Then, for any $\epsilon>0$ and finite subset $\mathcal{F} \subset C(X)$, there is a unital monomorphism $\phi: C \rightarrow A$ such that

$$
[\phi]=\kappa \text { and } \tau \circ \phi(f)=\gamma(f)(\tau) \text { for all } \tau \in T(A) .
$$

Proof. It is clear that the case that $C=M_{k}(C(X))$ follows from 4.5 immediately. For the general case, there is an integer $d \geq 1$ and a projection $p \in M_{d}(C)$ such that $p M_{d}(C) p \cong M_{m}(C(X))$ for some integer $m \geq 1$. Thus the general case is reduced to the case that $C=M_{m}(C(X))$.

Theorem 5.2. Let $C$ be a unital $A H$-algebra and let $A$ be a unital simple $C^{*}$-algebra with $T R(A)=0$. Suppose that $\kappa \in \mathrm{KL}_{e}(C, A)^{++}$. Suppose also that there is a unital strictly positive linear map $\gamma: C_{s, a} \rightarrow \operatorname{Aff}(T(A))$ which is compatible with $\kappa$. Then there is a monomorphism $\phi: C \rightarrow A$ such that

$$
\begin{aligned}
{[\phi] } & =\kappa \text { in } \operatorname{KL}(C, A) \text { and } \\
\tau \circ \phi(c) & =\gamma(c)(\tau)
\end{aligned}
$$

for all $c \in C_{\text {s.a. }}$ and $\tau \in T(A)$.

Proof. We may write $C=\overline{\cup_{n=1}^{\infty} C_{n}}$, where $C_{n}=P_{n} M_{k}\left(C\left(X_{n}\right)\right) P_{n}$, where $X_{n}$ is a compact subset of a finite CW complex and $P_{n} \in M_{k}\left(C\left(X_{n}\right)\right)$ is a projection. We may also assume that $1_{C_{n}}=1_{C}$ for all $n$. Denote by $\imath_{n}: C_{n} \rightarrow C$ the embedding, $n=1,2, \ldots$

Define

$$
\kappa_{n}=\kappa \circ\left[\imath_{n}\right] \text { and } \gamma_{n}=\gamma \circ\left(\imath_{n}\right)_{\sharp}
$$

$n=1,2, \ldots$ Since $\imath_{n}$ is injective $\kappa_{n} \in K L_{e}\left(C_{n}, A\right)^{++}$and $\gamma_{n}$ is unital strictly positive. It is also clear that $\gamma_{n}$ is compatible with $\kappa_{n}$, since $\gamma$ is compatible with $\kappa$. It follows from 5.1 that there is a sequence of unital monomorphisms $\phi_{n}: C_{n} \rightarrow A$ such that

$$
\left[\phi_{n}\right]=\kappa_{n} \text { and } \tau \circ \phi_{n}(c)=\gamma_{n}(c)(\tau)
$$

for all $c \in C_{\text {s.a. }}$ and $\tau \in T(A)$.

Let $\left\{\mathcal{F}_{n}\right\}$ be an increasing sequence of finite subsets of $C$ whose union is dense in $C$. By passing to a subsequence, if necessary, without loss of generality, we may assume that $\mathcal{F}_{n} \subset C_{n}$.

It follows (from 2.3.13 of [9], for example) that there is, for each $n$, a unital completely positive linear map $L_{n}: C \rightarrow A$ such that

$$
L_{n} \approx_{1 / 2^{n+1}} \phi_{n} \circ \imath_{n} \text { on } \mathcal{F}_{n} .
$$

It follows from Lemma 5.1, by passing to a subsequence again and by applying (e 5.71), there is a sequence of unitaries $u_{n}$ and a subsequence of $\{k(n)\}$ such that

$$
\text { ad } u_{n} \circ L_{k(n+1)} \approx_{1 / 2^{n}} L_{k(n)} \text { on } \mathcal{F}_{n},
$$

$n=1,2, \ldots$. Define $\psi_{1}=L_{1}, \psi_{n+1}=\operatorname{ad} u_{n} \circ L_{n+1}, n=1,2, \ldots$. Note that $\left\{\psi_{n}(c)\right\}$ is a Cauchy sequence in $A$ for each $c \in \mathcal{F}_{m}$. Define $h(c)=\lim _{n \rightarrow \infty} \psi_{n}(c)$. It is easy to see that $h$ gives a unital homomorphism from $C$ into $A$. Moreover, for each $x \in \cup_{n=1}^{\infty} \mathcal{F}_{n}$,

$$
h(x)=\lim _{n \rightarrow \infty} \operatorname{ad} u_{n} \circ \phi_{k(n)} \circ \imath_{k(n)} \circ \cdots \circ \imath_{n}(x) .
$$


Since each $\phi_{n}$ is injective, it follows that $h$ is a monomorphism. From (e 5.74) and (e 5.71), we have

$$
[h]=\kappa \text { as well as } \tau \circ h(c)=\gamma(c)(\tau)
$$

for all $c \in C_{\text {s.a. }}$ and $\tau \in T(A)$.

Corollary 5.3. Let $X$ be a compact metric space and let $A$ be a unital simple $C^{*}$-algebra with tracial rank zero. Suppose that $\kappa \in \mathrm{KL}_{e}(C(X), A)^{++}$. Suppose also that there is a unital strictly positive linear map $\gamma: C_{s, a} \rightarrow \operatorname{Aff}(T(A))$ which is compatible with $\kappa$. Then there is a monomorphism $\alpha: C \rightarrow A$ such that

$$
\begin{aligned}
{[\alpha] } & =\kappa \text { in } \mathrm{KL}(C(X), A) \text { and } \\
\tau \circ \phi(c) & =\gamma(c)(\tau)
\end{aligned}
$$

for all $c \in C(X)_{\text {s.a. }}$ and $\tau \in T(A)$.

Example 5.4. Let $X=\left\{\frac{-1}{n}: n \in \mathbb{N}\right\} \cup[0,1] \cup\left\{1+\frac{1}{n}: n \in \mathbb{N}\right\} \subset[-1,2]$. Put $C=C(X)$. Then

$$
K_{0}(C(X))=C(X, \mathbb{Z}) .
$$

Take two sequences of positive rational numbers $\left\{a_{n}\right\}$ and $\left\{b_{n}\right\}$ such that $\sum_{n=1}^{\infty} a_{n}=1-\sqrt{2} / 2$ and $\sum_{n=1}^{\infty}=\sqrt{2} / 2$.

Define a unital positive linear functional $F: C(X) \rightarrow \mathbb{R}$ as follows:

$$
F(f)=\sum_{n \in \mathbb{N}} a_{n} f\left(\frac{-1}{n}\right)+\sum_{n \in \mathbb{N}} b_{n} f\left(\frac{1}{n}\right) \text { for all } f \in C(X) .
$$

Let $D_{0}=F(C(X, \mathbb{Z}))$. Note that, if $S$ is a clopen subset which does not contain $[0,1]$, then $F(S) \in \mathbb{Q}$. If $S \supset[0,1]$, Then

$$
F(S)=1-F\left(S_{1}\right)
$$

for some clopen subset $S_{1} \subset X$ which does not intersect with $[0,1]$. It follows that $D_{0} \subset \mathbb{Q}$.

This gives a unital positive linear map $F_{*}: C(X, \mathbb{Z}) \rightarrow \mathbb{Q}$. Let $p \in C(X)$ be a projection whose support $\Omega$ has a non-empty intersection with $[0,1]$. Since $\Omega$ is clopen, $\Omega \supset[0,1]$. It follows that there is $N \geq 1$ such that $\frac{1}{k} \in \Omega$ for $|k| \geq N$. It follows that

$$
F(p) \geq \sum_{|k| \geq N} \frac{1}{2^{|k|+1}}>0 .
$$

From this one sees that $F_{*}$ is strictly positive.

Let $A$ be a unital simple AF-algebra with

$$
\left(K_{0}(A), K_{0}(A),\left[1_{A}\right]\right)=\left(\mathbb{Q}, \mathbb{Q}_{+}, 1\right) .
$$

There is an element $\kappa \in K L(C(X), A)$ such that

$$
\left.\kappa\right|_{K_{0}(C(X))}=F_{*} .
$$

Thus $\kappa\left(K_{0}(C(X))_{+} \backslash\{0\}\right) \subset K_{0}(A)_{+} \backslash\{0\}$. In other words, $\kappa \in K L_{e}(C, A)^{++}$.

Suppose that $\gamma: C_{\text {s.a }} \rightarrow \operatorname{Aff}(T(A))=\mathbb{R}$ is unital and positive such that

$$
\gamma(\check{p})(\tau)=\tau(\kappa([p]))
$$


for all projections $p \in C$ and $\tau \in T(A)$. Consider a positive continuous function $f \in C(X)$ with $0 \leq f \leq 1$ whose support $S$ is an open subset of $(0,1)$. Consider projection $p_{n}(t)=0$ if $t \notin[-1 / n, 1+1 / n] \cap X$ and $p_{n}(t)=1$ if $t \in[-1 / n, 1+1 / n] \cap X$. Then

$$
f \leq p_{n}, \quad n=1,2, \ldots
$$

It follows that, for all $\tau \in T(A)$,

$$
\begin{aligned}
\gamma(\check{f})(\tau) & \leq \gamma\left(\check{p}_{n}\right)(\tau) \\
& <\sum_{|k| \geq n}\left(a_{k}+b_{k}\right) \rightarrow 0
\end{aligned}
$$

as $|n| \rightarrow \infty$. It follows that

$$
\gamma(\check{f})(\tau)=0 \text { for all } \tau \in T(A) .
$$

This shows that $\gamma$ is not strictly positive.

In particular, there is no unital monomorphism $\phi: C(X) \rightarrow A$ such that $[\phi]=\kappa$.

How about homomorphisms? Suppose that there exists a unital homomorphism $h: C(X) \rightarrow$ $A$ such that $[h]=\kappa$. Let $f \in C(X)_{+}$be so that its support is contained in $[0,1]$. Then, as shown above, $\tau(h(f))=0$ for $\tau \in T(A)$. Since $A$ is simple, this implies that $h(f)=0$. It is then easy to see that

$$
\text { ker } h=\left\{f \in C(X):\left.f\right|_{X \backslash(0,1)}=0\right\} .
$$

Thus $C /$ ker $h \cong C(Y)$, where $Y=X \backslash(0,1)$. Let $\phi: C(Y) \rightarrow A$ be the unital homomorphism induced by $h$. Then $\phi$ is a monomorphism. Let

$$
Y_{1}=\{1+1 / n: n \in \mathbb{N}\} \cup\{1\} \text { and } Y_{2}=\{-1 / n: n \in \mathbb{N}\} \cup\{0\} .
$$

Then $Y_{1}$ and $Y_{2}$ are clopen subsets of $Y$. Let $p_{i}$ be the projection corresponding to $Y_{i}, i=1,2$. Then

$$
\tau\left(p_{1}\right) \geq \sum_{n=1}^{\infty} b_{n}=1-\sqrt{2} / 2 \text { and } \tau\left(p_{2}\right) \geq \sum_{n=1}^{\infty} a_{n}=\sqrt{2} / 2
$$

for $\tau \in T(A)$. Since $\tau\left(p_{1}\right)+\tau\left(p_{2}\right)=1$, it follows that

$$
\tau\left(p_{1}\right)=1-\sqrt{2} / 2 \text { and } \tau\left(p_{2}\right)=\sqrt{2} / 2 .
$$

This is impossible since $K_{0}(A)=\mathbb{Q}$.

From this we arrive at the following conclusion:

Proposition 5.5. There are compact metric spaces $X$ with dimension one, unital simple AFalgebras $A$ with unique tracial states and $\kappa \in K L_{e}(C, A)^{++}$which has no strictly positive affine map from $\operatorname{Aff}(T(C(X))$ to $\operatorname{Aff}(T(A))$ compatible with $\kappa$.

Furthermore, there is no unital homomorphism $\phi: C(X) \rightarrow A$ such that $[\phi]=\kappa$ in $K L(C, A)$.

Definition 5.6. Let $C$ be a unital AH-algebra which admits a faithful tracial state and let $A$ be a unital simple $C^{*}$-algebra with $T(A) \neq \emptyset$.

Denote by $K L T(C, A)^{++}$the set of pairs $(\kappa, \lambda)$ where $\kappa \in K L(C, A)^{++}$with $\kappa\left(\left[1_{C}\right]\right)=\left[1_{A}\right]$ and $\lambda: T(A) \rightarrow T_{\mathrm{f}}(C)$ which is compatible with $\kappa$, i.e., $\lambda(\tau)(p)=\tau(\kappa([p])$ for all projections $p \in M_{\infty}(C)$ and for all $\tau \in T(A)$.

Denote by $\operatorname{Mon}_{a u}^{e}(C, A)$ the set of approximately unitary equivalent classes of unital monomorphisms from $C$ into $A$. 
To conclude this note, combing the previous result in ?? (see [3.1) and 5.2, we state the following:

Theorem 5.7. Let $C$ be a unital AH-algebra which admits a faithful tracial state and let $A$ be a unital separable simple $C^{*}$-algebra with $T R(A)=0$. Then map

$$
\Lambda: \operatorname{Mon}_{a u}^{e}(C, A) \rightarrow K L T(C, A)^{++}
$$

defined by $\phi \mapsto\left([\phi], \phi_{T}\right)$ is bijective.

\section{References}

[1] M. Dădărlat and T. A. Loring, A universal multicoefficient theorem for the Kasparov groups, Duke Math. J. 84 (1996), 355-377.

[2] M. Dădărlat and T. A. Loring, The K-theory of abelian subalgebras of AF algebras, J. Reine Angew. Math. 432 (1992), 39-55.

[3] G. A. Elliott and T. Loring, AF embeddings of $C\left(\mathbf{T}^{2}\right)$ with a prescribed $K$-theory, J. Funct. Anal. 103 (1992), 1-25.

[4] G. Gong and H. Lin, Classification of homomorphisms from $C(X)$ to simple $C^{*}$-algebras of real rank zero, Acta Math. Sin. (Engl. Ser.) 16 (2000), 181-206.

[5] G. Gong and H. Lin, Almost multiplicative morphisms and K-theory, Internat. J. Math. 11 (2000), 983-1000.

[6] L. Li, $C^{*}$-algebra homomorphisms and KK-theory, K-Theory 18 (1999), 161-172.

[7] H. Lin, Tracially AF $C^{*}$-algebras, Trans. Amer. Math. Soc. 353 (2001), 693-722.

[8] H. Lin, Tracial topological ranks of $C^{*}$-algebras, Proc. London Math. Soc., 83 (2001), 199-234.

[9] H. Lin, An introduction to the classification of amenable $C^{*}$-algebras, World Scientific Publishing Co., Inc., River Edge, NJ, 2001. xii+320 pp. ISBN: 981-02-4680-3.

[10] H. Lin, Embedding an AH-algebra into a simple $C^{*}$-algebra with prescribed $K K$-data. $K$ Theory 24 (2001), 135-156.

[11] H. Lin Classification of homomorphisms and dynamical systems, Trans. Amer. Math. Soc. 359 (2007), 859-895.

[12] H. Lin Approximate Homotopy of Homomorphisms from $C(X)$ into a Simple $C^{*}$-algebra, preprint (arXiv:math/0612125).

[13] H. Lin AF-embedding of crossed products of AH-algebras by $\mathbb{Z}$ and asymptotic AFembedding, Indiana Math. J., to appear (arXiv:math/0612529).

[14] S. Zhang, A Riesz decomposition property and ideal structure of multiplier algebras, J. Operator Theory 24 (1990), 209-225.

[15] S. Zhang, Matricial structure and homotopy type of simple $C^{*}$-algebras with real rank zero, J. Operator Theory 26 (1991), 283-312. 